Bangladesh J. Plant Taxon. 17(1): 9-22, 2010 (June)

(C) 2010 Bangladesh Association of Plant Taxonomists

\title{
ANGIOSPERM DIVERSITY OF LAWACHARA NATIONAL PARK (BANGLADESH): A PRELIMINARY ASSESSMENT
}

\author{
Mohammad Zashim Uddin ${ }^{1}$ And Md. Abul Hassan \\ Department of Botany, University of Dhaka, Dhaka 1000, Bangladesh
}

Keywords: Preliminary assessment; Angiosperm diversity; Lawachara National Park.

\begin{abstract}
The present article focuses on an inventory of angiosperm diversity of Lawachara National Park. Using traditional taxonomic techniques data have been collected from the Park during January to October 2008. In this preliminary assessment, a total of 374 angiosperm species including cultivated ones have been catalogued under 84 families. Nineteen threatened plant species have also been recognized in the Park.
\end{abstract}

\section{Introduction}

Lawachara National Park under Kamalganj upazila of Maulvi Bazar district is a part of West Bhanugach reserve forest, which was declared reserve in early nineteenth century as per the Forest Act 1878, the Assam Forest Manual 1898 and the Forest Act 1927. The Park is located nearly $160 \mathrm{~km}$ northeast of Dhaka and approximately $60 \mathrm{~km}$ south of Sylhet city. It lies between $24^{\circ} 30^{\prime}-24^{\circ} 32^{\prime} \mathrm{N}$ latitude and $91^{\circ} 37^{\prime}-91^{\circ} 39^{\prime} \mathrm{E}$ longitude. A part of the reserve forest was declared as a National Park in 1996 having a total area of 1250 ha (Green, 1990; Canonizado and Rahman, 1998; Riadh, 2007; Ahsan, 2007). Present forest types of Lawachara are a combination of planted exotic species and mixed forest with a deciduous canopy and an evergreen understory (Ahsan, 2000). The forest originally supported an indigenous vegetation cover of mixed tropical evergreen type (Alam, 1998).

The topography of Lawachara National Park is undulating, with slopes and hillocks that range from 10 to $50 \mathrm{~m}$ in elevation (Rizvi, 1970; Riadh, 2007). These hillocks are scattered and interspersed with numerous streams that flow through the forest. The hills are composed of upper tertiary rocks in which sand stone largely predominates (Ahmad, 1970; Stevens, 1986) along with siltstones and mudstones, locally altered to slates and shales. The significant soils in the hills of Maulvi Bazar belong to Ramgarh and Rangamati series on Dupitila formation (Stevens, 1986). Soils of the Park are generally sandy loam and the rest are mostly clayey loam (Ahmad, 1970). The area enjoys a moist tropical climate characterized by a period of high precipitation from April to September and five months of relatively dry period from November to March.

${ }^{1}$ Corresponding author. E-mail: zashim07@yahoo.com 
In Bangladesh, assessment of plant diversity of the forests of different protected areas has already been started. Khan et al. (1994), Rahman and Hassan (1995), Uddin et al. (1998), Uddin and Rahman (1999), Khan and Huq (2001) and Uddin and Hassan (2004) have so far published floristic works but no such work was carried out for Lawachara National Park. The values of different aspects of this Park is now realized both by local and international communities. One international organization USAID and Nishorgo Support Project in collaboration with Forest Department have been working in the Park to establish co-management strategy for the conservation of biodiversity and sustainable development. For the sake of management of biodiversity, assessment of plant diversity especially angiosperm diversity as major component of the park, is very essential that will provide baseline information on which action plan can be made. The present study has been undertaken to make an inventory of angiosperm diversity of Lawachara National Park.

\section{Materials and Methods}

Six field visits, each lasted for five days have been made to the area during January 2008 to October 2008. Each section of this spectacular National Park was carefully combed to assure all species in the list including those that were scattered or infrequent. Special effort was made to locate the species already listed as threatened or endangered in the country. Botanical specimens were collected and field identifications were confirmed back at Dhaka University Herbarium (presently known as Salar Khan Herbarium). Voucher specimens were preserved in the same Herbarium. Woody flora of Sylhet (Alam, 1988) and flora of Rema-Kalenga wildlife sanctuary (Uddin and Hassan, 2004) were also consulted for the identification of specimens.

\section{Results and Discussion}

Based on this study, a preliminary list of angiosperm diversity of the Lawachara National Park was made that includes 374 species under 264 genera and 84 families. For each species scientific name, Bengali name (when available) and family are provided (Table 1). Of 374 species recorded here, herbs are represented by 148 , shrubs by 71 , trees by 90 and climbers by 65 species. Nineteen species listed as threatened in the Red Data Book of Vascular Plants of Bangladesh (Khan et al., 2001) have been located in this Park. These are Ammomum aromaticum, Aquillaria agallocha, Bombax insigne, Calamus guruba, Cymbidium aloifolium, Desmos longiflorus, Globba multiflora, Hedychium coccineum, Hedychium thyrsiforme, Holigarna longifolia, Hydnocarpus kurzii, Mangifera sylvatica, Phrynium imbricatum, Pinanga gracilis, Pterospermum semisagittatum, Rauvolfia serpentina, Steudnera colocasioides, Taxillus thelocarpa and Terminalia citrina. One species of gymnosperm (Gnetum oblongum) and one species of tree fern (Cyathea gigantea) listed as endangered in Bangladesh were also located in the Park during this survey. 
Table 1. Diversity of angiosperm species of Lawachara National Park $(h=h e r b, s=s h r u b, t=t r e e$, c = climber)

\begin{tabular}{|c|c|c|c|c|}
\hline S1. No & Species name & Bengali name & Family & Habit \\
\hline 1 & Acacia auriculiformis A. Cunn. ex Benth. & Akashmoni & Mimosaceae & $\mathrm{t}$ \\
\hline 2 & A. concinna DC. & Kuchui & Mimosaceae & $\mathrm{s}$ \\
\hline 3 & A. mangium Willd. & - & Mimosaceae & $\mathrm{t}$ \\
\hline 4 & Acamphae premorsa (Roxb.) Blatter \& Mcann & - & Orchidaceae & $\mathrm{h}$ \\
\hline 5 & Achyranthes aspera L. & Apang & Amaranthaceae & $\mathrm{h}$ \\
\hline 6 & Actinodaphnae angustifolia Nees & - & Lauraceae & $\mathrm{t}$ \\
\hline 7 & Adhatoda zeylanica Medikus & Bhasak & Acanthaceae & $\mathrm{h}$ \\
\hline 8 & Aerva sanguinolenta (L.) Bl. & Lalapang & Amaranthaceae & $\mathrm{h}$ \\
\hline 9 & Ageratum conyzoides L. & Fulkuri & Asteraceae & $\mathrm{h}$ \\
\hline 10 & Albizia odoratissima Benth. & Kalokoroi & Mimosaceae & $\mathrm{t}$ \\
\hline 11 & A. procera (Roxb.) Benth. & Koroi & Mimosaceae & $\mathrm{t}$ \\
\hline 12 & Allophyllus cobbe B1. & - & Sapindaceae & $\mathrm{h}$ \\
\hline 13 & Alocasia cuculata (Lour.) G. Don & - & Araceae & $\mathrm{h}$ \\
\hline 14 & A. indica Scott. & Mankachu & Araceae & $\mathrm{h}$ \\
\hline 15 & Alpinia malaccensis (Burm. f.) Rosc. & Deotara & Zingiberaceae & $\mathrm{h}$ \\
\hline 16 & Alstonia scholaris L. & Chatim & Apocynaceae & $\mathrm{t}$ \\
\hline 17 & Alternanthera sessilis (L.) R. Br. ex DC. & Chanchi & Amaranthaceae & $\mathrm{h}$ \\
\hline 18 & Ammomum aromaticum Roxb. & Morangelachi & Zingiberaceae & $\mathrm{h}$ \\
\hline 19 & A. corynostachyum Wall. & - & Zingiberaceae & $\mathrm{h}$ \\
\hline 20 & A. dealbatum Roxb. & - & Zingiberaceae & $\mathrm{h}$ \\
\hline 21 & A. uliginosum Koen. & - & Zingiberaceae & $\mathrm{h}$ \\
\hline 22 & Amoora wallichii King & Lali & Meliaceae & $\mathrm{t}$ \\
\hline 23 & Amorphophalus campanulatus B1. & Olkachu & Araceae & $\mathrm{h}$ \\
\hline 24 & Ampelygonum chinense (L.) Lindley & - & Polygonaceae & $\mathrm{h}$ \\
\hline 25 & Anacardium occidentalis L. & Kajubadam & Anacardiaceae & $\mathrm{t}$ \\
\hline 26 & Ananas sativus Schult. f. & Anaros & Bromeliaceae & $\mathrm{h}$ \\
\hline 27 & Anisomeles indica (L.) Kuntze & Gobura & Lamiaceae & $\mathrm{h}$ \\
\hline 28 & Anthocephalus chinensis (Lamk.) A. Rich ex Walp. & Kadam & Rubiaceae & $\mathrm{t}$ \\
\hline 29 & Antidesma ghaesemblia Gaertn. & - & Euphorbiaceae & $\mathrm{s}$ \\
\hline 30 & A. roxburghii Wall. & - & Euphorbiaceae & $\mathrm{s}$ \\
\hline 31 & Aphanamixis polystachya (Wall.) Parker & Pitraj & Meliaceae & $\mathrm{t}$ \\
\hline 32 & Aphania danura (Roxb.) Rodlk. & Danura & Sapindaceae & $\mathrm{s}$ \\
\hline 33 & Aporosa dioica (Roxb.) Muell.-Arg. & Patakharolla & Euphorbiaceae & $\mathrm{t}$ \\
\hline 34 & A. oblonga (Wall.) Muell.-Arg. & - & Euphorbiaceae & $\mathrm{t}$ \\
\hline 35 & Aquillaria agallocha Roxb. & Agar & Thymeliaceae & $\mathrm{t}$ \\
\hline 36 & Ardisia colorata Roxb. & - & Myrsinaceae & $\mathrm{s}$ \\
\hline 37 & A. paniculata Roxb. & - & Myrsinaceae & $\mathrm{s}$ \\
\hline 38 & A. solanacea Roxb. & Banjam & Myrsinaceae & $\mathrm{s}$ \\
\hline 39 & Areca catechu L. & Supari & Arecaceae & $\mathrm{t}$ \\
\hline 40 & Argyria capitiformis (Poir.) Oostr. & - & Convolvulaceae & $\mathrm{c}$ \\
\hline
\end{tabular}


Table 1 Contd.

\begin{tabular}{|c|c|c|c|c|}
\hline \multicolumn{2}{|c|}{ Sl. No. Species name } & \multirow{2}{*}{$\begin{array}{l}\text { Bengali name } \\
-\end{array}$} & \multirow{2}{*}{$\begin{array}{r}\text { Family } \\
\text { Orchidaceae }\end{array}$} & \multirow{2}{*}{$\frac{\text { Habit }}{\mathrm{h}}$} \\
\hline 41 & Arides odorata Lour. & & & \\
\hline 42 & Aristolochia tagala Cham. & - & Aristolochiaceae & $\mathrm{c}$ \\
\hline 43 & Artocarpus heterophyllus Lamk. & Kanthal & Moraceae & $\mathrm{t}$ \\
\hline 44 & A. chaplasha Roxb. & Chapalish & Moraceae & $\mathrm{t}$ \\
\hline 45 & A. lakucha Roxb. & Deua & Moraceae & $\mathrm{t}$ \\
\hline 46 & Arundinella bengalensis (Spreng.) Druce & - & Poaceae & $\mathrm{h}$ \\
\hline 47 & Axonopus compressus (Swartz.) P. Beauv. & - & Poaceae & $\mathrm{t}$ \\
\hline 48 & Azadirachta indica A. Juss. & Neem & Meliaceae & $\mathrm{t}$ \\
\hline 49 & Baccaurea ramiflora Lour. & Lotkon & Euphorbiaceae & $\mathrm{t}$ \\
\hline 50 & Bambusa balcooa Roxb. & Barak bans & Poaceae & $\mathrm{s}$ \\
\hline 51 & B. bambos (L.) Voss & Kanta bans & Poaceae & $\mathrm{s}$ \\
\hline 52 & B. polymorpha Munro & Parua & Poaceae & $\mathrm{s}$ \\
\hline 53 & B. tulda Roxb. & Mitinga & Poaceae & $\mathrm{s}$ \\
\hline 54 & B. vulgaris Schrad. ex Wendl. & Baijja & Poaceae & $\mathrm{t}$ \\
\hline 55 & Begonia barbata Wall. ex A. DC. & - & Begoniaceae & $\mathrm{h}$ \\
\hline 56 & B. roxburghii (Miq.) DC. & - & Begoniaceae & $\mathrm{h}$ \\
\hline 57 & Bischofia javanica B1. & Kanjalbhady & Euphorbiaceae & s \\
\hline 58 & Bombax ceiba L. & Shimul & Bombacaceae & $\mathrm{t}$ \\
\hline 59 & B. insigne Wall. & Bonshimul & Bombacaceae & $\mathrm{t}$ \\
\hline 60 & Borreria articularis (L. f.) Williams & - & Rubiaceae & $\mathrm{h}$ \\
\hline 61 & Breynia patens Benth. & Kakro & Euphorbiaceae & $\mathrm{s}$ \\
\hline 62 & Bridelia stipularis (L.) Bl. & - & Euphorbiaceae & $\mathrm{c}$ \\
\hline 63 & Brownlowia eleta Roxb. & - & Sterculiaceae & $\mathrm{t}$ \\
\hline 64 & Bulbophyllum lilacinum Ridley & - & Orchidaceae & $\mathrm{h}$ \\
\hline 65 & Bursera serrata Wall. ex Colobr. & Neul & Burseraceae & $\mathrm{t}$ \\
\hline 66 & Butea monosperma (Lamk.) Taub. & Palash & Fabaceae & $\mathrm{t}$ \\
\hline 67 & Buttnera pilosa Roxb. & - & Sterculiaceae & $\mathrm{c}$ \\
\hline 68 & Caesalpinia bonduc Roxb. & Nata & Caesalpiniaceae & $\mathrm{c}$ \\
\hline 69 & Calamus guruba Buch.-Ham. ex Mart. & Jalibet & Arecaceae & $\mathrm{c}$ \\
\hline 70 & Calotropis gigantea (L.) R. Br. & Akonda & Asclepiadaceae & $\mathrm{s}$ \\
\hline 71 & Calycarpa arborea Roxb. & Bormala & Verbenaceae & $\mathrm{t}$ \\
\hline 72 & C. lanciolaria Roxb. & - & Verbenaceae & $\mathrm{s}$ \\
\hline 73 & Carex jenkensianus Boott. & - & Cyperaceae & $\mathrm{h}$ \\
\hline 74 & Carya arborea Roxb. & Kumbi & Lecythidaceae & $\mathrm{t}$ \\
\hline 75 & Caryota urens L. & Golsagu & Arecaceae & $\mathrm{t}$ \\
\hline 76 & Cassia hirsuta L. & - & Caesalpiniaceae & $\mathrm{h}$ \\
\hline 77 & C. occidentalis L. & Eski & Caesalpiniaceae & $\mathrm{s}$ \\
\hline 78 & C. sophera L. & Kalkesunde & Caesalpiniaceae & $\mathrm{h}$ \\
\hline 79 & C. tora $\mathrm{L}$ & - & Caesalpiniaceae & $\mathrm{h}$ \\
\hline 80 & Castanopsis tribuloides A. DC. & Khami & Fagaceae & $\mathrm{t}$ \\
\hline
\end{tabular}


Table 1 Contd.

\begin{tabular}{|c|c|c|c|c|}
\hline S1. No & Species name & Bengali name & Family & Habit \\
\hline 81 & Casuarina littorea L. & Jhau & Casuarinaceae & $\mathrm{t}$ \\
\hline 82 & Cayratia japonica (Thunb.) Gagnep. & - & Vitaceae & $\mathrm{c}$ \\
\hline 83 & Centella asiatica Urban & Thankuni & Apiaceae & $\mathrm{h}$ \\
\hline 84 & Centotheca lappacea (L.) Desv. & - & Poaceae & $\mathrm{h}$ \\
\hline 85 & Ceriscoides campanulata (Roxb.) Tirveng. & - & Rubiaceae & $\mathrm{s}$ \\
\hline 86 & Chassalia curviflora (Wall.) Thw. & - & Rubiaceae & $\mathrm{h}$ \\
\hline 87 & Chlorophora excelsa (Welw.) Benth. & - & Moraceae & $\mathrm{t}$ \\
\hline 88 & Chrysopogon aciculatus (Retz.) Trin. & Premkanta & Poaceae & $\mathrm{h}$ \\
\hline 89 & Chukrasia tabularis A. Juss. & Chikrasi & Meliaceae & $\mathrm{t}$ \\
\hline 90 & Cissus adnata Roxb. & Aliangalata & Vitaceae & $\mathrm{c}$ \\
\hline 91 & Citrus grandis (L.) Osb. & Jambura & Rutaceae & $\mathrm{s}$ \\
\hline 92 & Clausena heptaphylla (Roxb.) Wight \& Arn. & Pomkafur & Rutaceae & $\mathrm{h}$ \\
\hline 93 & Clerodendrum serratum (L.) Moon. & Barangi & Verbenaceae & $\mathrm{h}$ \\
\hline 94 & C. viscosum Vent. & Bhant & Verbenaceae & $\mathrm{h}$ \\
\hline 95 & Cnesmone javanica B1. & - & Euphorbiaceae & $\mathrm{s}$ \\
\hline 96 & Colocasia esculenta (L.) Schott & Kachu & Araceae & $\mathrm{h}$ \\
\hline 97 & Combretum acuminatum Roxb. & Patuinia & Combretaceae & $\mathrm{c}$ \\
\hline 98 & C. apetalum Wall. & - & Combretaceae & $\mathrm{c}$ \\
\hline 99 & C. latifolium Bl. & Baulata & Combretaceae & $\mathrm{c}$ \\
\hline 100 & C. punctatum B1. & - & Combretaceae & $\mathrm{c}$ \\
\hline 101 & Commelina benghalensis L. & Kanchira & Commelinaceae & $\mathrm{h}$ \\
\hline 102 & C. erecta $\mathrm{L}$. & - & Commelinaceae & $\mathrm{h}$ \\
\hline 103 & Costus speciosa (Koening) Smith & Kura & Costaceae & $\mathrm{h}$ \\
\hline 104 & Crotalaria juncea $\mathrm{L}$. & Shonpat & Fabaceae & $\mathrm{h}$ \\
\hline 105 & Croton caudatus Geisel. & Sabarjala & Euphorbiaceae & $\mathrm{s}$ \\
\hline 106 & Curculigo orchioides Gaer. & Talmuli & Liliaceae & $\mathrm{h}$ \\
\hline 107 & C. recurvata Dryand. & Bidipata & Liliaceae & $\mathrm{h}$ \\
\hline 108 & Curcuma zedoaria (Christm.) Rosc. & Shathi & Zingiberaceae & $\mathrm{h}$ \\
\hline 109 & Cyclea peltata Hook. f. et Thom. & - & Menispermaceae & $\mathrm{c}$ \\
\hline 110 & Cymbidium aloifolium (L.) Sw. & - & Orchidaceae & $\mathrm{h}$ \\
\hline 111 & Cynodon dactylon L. & Durba & Poaceae & $\mathrm{h}$ \\
\hline 112 & Cyperus cyperoides (L.) O. Kuntze & - & Cyperaceae & $\mathrm{h}$ \\
\hline 113 & C. difformis L. & Bethua & Cyperaceae & $\mathrm{h}$ \\
\hline 114 & C. exaltatus Retz & - & Cyperaceae & $\mathrm{h}$ \\
\hline 115 & C. pilosus Vahl & - & Cyperaceae & $\mathrm{h}$ \\
\hline 116 & C. rotundus $\mathrm{L}$. & Mutha & Cyperaceae & $\mathrm{h}$ \\
\hline 117 & Daemonorops jenkensianas (Griff.) Mart. & Golakbet & Arecaceae & $\mathrm{c}$ \\
\hline 118 & Dalbergia stipularis Roxb. \& Baker & Dadbari & Fabaceae & $\mathrm{c}$ \\
\hline 119 & D. volubilis Roxb. & Ankilata & Fabaceae & $\mathrm{s}$ \\
\hline 120 & Dehaasia kurzii King ex Hook. f. & Modonmosta & Lauraceae & $\mathrm{t}$ \\
\hline 121 & Dendrobium aphyllum (Roxb.) Fisch. & - & Orchidaceae & $\mathrm{h}$ \\
\hline 122 & D. lindleyi Steud. & - & Orchidaceae & $\mathrm{h}$ \\
\hline
\end{tabular}


Table 1 Contd.

\begin{tabular}{|c|c|c|c|c|}
\hline Sl. N & Species name & Bengali name & Family & Habit \\
\hline 123 & Derris elegans Benth. & - & Fabaceae & $\mathrm{c}$ \\
\hline 124 & D. scandens Benth. & Kamirialata & Fabaceae & $\mathrm{c}$ \\
\hline 125 & Desmodium motorium (Houtt.) Merril. & Loncharal & Fabaceae & $\mathrm{h}$ \\
\hline 126 & D. pulchellum (L.) Benth. & Jutasalpani & Fabaceae & $\mathrm{s}$ \\
\hline 127 & Desmos chinensis Lour. & - & Annonaceae & $\mathrm{c}$ \\
\hline 128 & D. longiflorus (Roxb.) Safford & - & Annonaceae & $\mathrm{t}$ \\
\hline 129 & Digitaria adscendens (HBK) Henr. & - & Poaceae & $\mathrm{h}$ \\
\hline 130 & Dillenia pentagyna Roxb. & Hargoza & Dilleniaceae & $\mathrm{t}$ \\
\hline 131 & D. scabrella (D. Don) Roxb. ex Wall. & Hargoza & Dilleniaceae & $\mathrm{t}$ \\
\hline 132 & Dioscorea belophylla (Prain) J. O. Voigt ex Haines & Shoraalu & Dioscoreaceae & $\mathrm{c}$ \\
\hline 133 & D. bulbifera L. & Ratal & Dioscoreaceae & $\mathrm{c}$ \\
\hline 134 & D. hispida Dennst. & - & Dioscoreaceae & $\mathrm{c}$ \\
\hline 135 & D. pentaphylla L. & Jhunihanalata & Dioscoreaceae & $\mathrm{c}$ \\
\hline 136 & D. tomentosa Koenig ex Spreng. & - & Dioscoreaceae & $\mathrm{c}$ \\
\hline 137 & D. triphylla Ham. & - & Dioscoreaceae & $\mathrm{c}$ \\
\hline 138 & D. trinerva Roxb. & - & Dioscoreaceae & $\mathrm{c}$ \\
\hline 139 & Diospyros montana Roxb. & Tamal & Ebenaceae & $\mathrm{t}$ \\
\hline 140 & Dipterocarpus turbinatus Gaertn. & Kaligarjan & Dipterocarpaceae & $\mathrm{t}$ \\
\hline 141 & Dracaena spicata Roxb. & Dracaena & Liliaceae & $\mathrm{h}$ \\
\hline 142 & Dysophyla auricularia Bl. & - & Lamiaceae & $\mathrm{h}$ \\
\hline 143 & Eclipta prostrata L. & Kesaraj & Asteraceae & $\mathrm{h}$ \\
\hline 144 & Elaeocarpus floribundus Bl. & Belphoi & Elaeocarpaceae & $\mathrm{t}$ \\
\hline 145 & E. robustus Roxb. & Jalpai & Elaeocarpaceae & $\mathrm{t}$ \\
\hline 146 & Eragrostis ciliaris (L.) R. Br. & - & Poaceae & $\mathrm{h}$ \\
\hline 147 & E. tenella (L.) P. Beauv. ex Roem. \& Schult. & - & Poaceae & $\mathrm{h}$ \\
\hline 148 & E. tenuifolia Hochst. ex Steud. & - & Poaceae & $\mathrm{h}$ \\
\hline 149 & E. uniloides (Retz.) Nees ex Steud. & - & Poaceae & $\mathrm{h}$ \\
\hline 150 & Eranthemum album Nees & Muralipata & Acanthaceae & $\mathrm{h}$ \\
\hline 151 & Erioglossum rubiginosum (Roxb.) B1. & Baraharina & Sapindaceae & $\mathrm{s}$ \\
\hline 152 & Ervatamia coronaria (Jacq.) Stapf. & Togor & Apocynaceae & $\mathrm{s}$ \\
\hline 153 & Erythrina ovalifolia Roxb. & Mandar & Fabaceae & $\mathrm{t}$ \\
\hline 154 & Eupatorium odoratum L. & Assamlata & Asteraceae & $\mathrm{s}$ \\
\hline 155 & Ficus benghalensis L. & Bot & Moraceae & $\mathrm{t}$ \\
\hline 156 & F. hirta Vahl & - & Moraceae & $\mathrm{s}$ \\
\hline 157 & F. hispida L. f. & Dumur & Moraceae & $\mathrm{h}$ \\
\hline 158 & F. irisiana Elm. & - & Moraceae & $\mathrm{c}$ \\
\hline 159 & F. racemosa $\mathrm{L}$. & Jagdumur & Moraceae & $\mathrm{t}$ \\
\hline 160 & F. ramentacea $\mathrm{Roxb}$. & - & Moraceae & $\mathrm{c}$ \\
\hline 161 & F. religiosa $\mathrm{L}$. & Assawatha & Moraceae & $\mathrm{t}$ \\
\hline 162 & F. retusa $\mathrm{L}$. & - & Moraceae & $\mathrm{s}$ \\
\hline 163 & Fimbristylis aestivalis (Retz) Vahl & - & Cyperaceae & $\mathrm{h}$ \\
\hline 164 & F. dichotoma (L.) Vahl & Baranirbishi & Cyperaceae & $\mathrm{h}$ \\
\hline
\end{tabular}


Table 1 Contd.

\begin{tabular}{|c|c|c|c|c|}
\hline S1. No & Species name & Bengali name & Family & Habit \\
\hline 165 & F. falcata (Vahl) Kunth & - & Cyperaceae & $\mathrm{h}$ \\
\hline 166 & Flacourtia indica (Burm. f.) Merr. & Paniala & Flacourtiaceae & $\mathrm{s}$ \\
\hline 167 & F. jangomus (Lour.) Raeusch & Lukluki & Flacourtiaceae & $\mathrm{s}$ \\
\hline 168 & Floscopa scandens Lour. & - & Commelinaceae & $\mathrm{h}$ \\
\hline 169 & Fuirena ciliaris (L.) Roxb. & - & Cyperaceae & $\mathrm{h}$ \\
\hline 170 & Garcinia cowa Roxb. & $\mathrm{Kau}$ & Clusiaceae & $\mathrm{t}$ \\
\hline 171 & G. pedunculata Roxb. & - & Clusiaceae & $\mathrm{t}$ \\
\hline 172 & G. xanthochymus Hook. f. ex T. Ander. & Dayphal & Clusiaceae & $\mathrm{s}$ \\
\hline 173 & Gardenia coronaria Ham. & Koinar & Rubiaceae & $\mathrm{s}$ \\
\hline 174 & Garuga pinnata Roxb. & Kharapat & Burseraceae & $\mathrm{t}$ \\
\hline 175 & Gouania laptostachya DC. & - & Rhamnaceae & $\mathrm{c}$ \\
\hline 176 & Gigantochloa andamanica (Kurz) Kurz & Kali & Poaceae & $\mathrm{s}$ \\
\hline 177 & Globba multiflora Wall. ex Baker & - & Zingiberaceae & $\mathrm{h}$ \\
\hline 178 & G. orixensis Roxb. & - & Zingiberaceae & $\mathrm{h}$ \\
\hline 179 & Glochidion multi-loculare (Roxb. ex Willd) Muell.-Arg. & Kakra & Euphorbiaceae & $\mathrm{s}$ \\
\hline 180 & Glycosmis arborea Roxb. & Datmajan & Rutacae & $\mathrm{s}$ \\
\hline 181 & Gmelina arborea Roxb. & Gamari & Verbenaceae & $\mathrm{t}$ \\
\hline 182 & Grangea madaraspatana (L.) Poir. & Nemuti & Asteraceae & $\mathrm{h}$ \\
\hline 183 & Grewia microcos L. & Assar & Tiliaceae & $\mathrm{s}$ \\
\hline 184 & Gymnopetalum cochinchinensis (Lour.) Kurz & - & Cucurbitaceae & $\mathrm{c}$ \\
\hline 185 & Gynostemma pentaphylla (Thunb.) Makino & - & Vitaceae & $\mathrm{c}$ \\
\hline 186 & Hedychium coccineum Buch.-Ham. ex Smith & Bhuiada & Zingiberaceae & $\mathrm{h}$ \\
\hline 187 & H. thyrsiforme Buch.-Ham. ex Smith & - & Zingiberaceae & $\mathrm{h}$ \\
\hline 188 & Hemidesmus indicus (L.) R. Br. & Anantamul & Asclepiadaceae & $\mathrm{c}$ \\
\hline 189 & Heteria rubens Benth. ex Hook. f. & - & Orchidaceae & $\mathrm{h}$ \\
\hline 190 & Heterophragma adenophyllum Seem. & - & Bignoniacae & $\mathrm{t}$ \\
\hline 191 & Hevea brasiliensis Muell.-Arg. & Rubber & Euphorbiaceae & $\mathrm{t}$ \\
\hline 192 & Holarrhena antidysenterica (L.) Wall. & Kurchi & Apocynaceae & s \\
\hline 193 & Holigarna longifolia Roxb. & Barola & Anacardiaceae & $\mathrm{t}$ \\
\hline 194 & Homalomena aromatica Schott. & Gandhabi & Araceae & $\mathrm{h}$ \\
\hline 195 & Hoya parasitica (Wall.) Wight & Pargacha & Asclepiadaceae & $\mathrm{c}$ \\
\hline 196 & Hydnocarpus kurzii (King) Warb. & Chaulmoogra & Flacourtiaceae & $\mathrm{t}$ \\
\hline 197 & Hypericum japonicum Thunb. & - & Hypericaceae & $\mathrm{h}$ \\
\hline 198 & Hyptis suaveolens (L.) Poit. & Tokma & Lamiaceae & $\mathrm{h}$ \\
\hline 199 & Ichnocarpus frutescens (L.) R. Br. & Shamalata & Apocynaceae & $\mathrm{c}$ \\
\hline 200 & Imperata cylindrica (L.) P. Beauv. & Ulu & Poaceae & $\mathrm{h}$ \\
\hline 201 & Ipomoea fistulosa Mart. ex Choisy & Dholkalmi & Convovulaceae & $\mathrm{s}$ \\
\hline 202 & Ixora arborea Roxb. ex Smith & Swetrangan & Rubiaceae & $\mathrm{s}$ \\
\hline 203 & I. javanica Roxb. ex Smith & - & Rubiaceae & $\mathrm{s}$ \\
\hline 204 & I. parviflora $\mathrm{Vahl}$ & Swetrangan & Rubiaceae & $\mathrm{s}$ \\
\hline 205 & Jasminum sambac Ait. & Beli & Oleaceae & $\mathrm{s}$ \\
\hline 206 & Justicia ganderusa L. & Nilnishinda & Acanthaceae & $\mathrm{s}$ \\
\hline
\end{tabular}


Table 1 Contd.

\begin{tabular}{|c|c|c|c|c|}
\hline Sl. N & Species name & Bengali name & Family & Habit \\
\hline 207 & Lagerstroemia indica L. & - & Lythraceae & $\mathrm{t}$ \\
\hline 208 & L. parviflora Roxb. & Sidha & Lythraceae & $\mathrm{t}$ \\
\hline 209 & L. speciosa (L.) Pers. & Jarul & Lythraceae & $\mathrm{t}$ \\
\hline 210 & Lannea coromandelica (Houtt.) Merr. & Jiga & Anacardiaceae & $\mathrm{t}$ \\
\hline 211 & Lantana camara L. & Lantana & Verbenaceae & $\mathrm{h}$ \\
\hline 212 & Laportia cranulata Gaud. & Agnichutra & Urticaceae & $\mathrm{c}$ \\
\hline 213 & Leea acuminata (Burm. f.) Merr. & - & Leeaceae & $\mathrm{h}$ \\
\hline 214 & L. aequata $\mathrm{L}$. & Kakjangha & Leeaceae & $\mathrm{h}$ \\
\hline 215 & L. crispa Willd. & - & Leeaceae & $\mathrm{s}$ \\
\hline 216 & Lepidagathis incurva D. Don & - & Acanthaceae & $\mathrm{h}$ \\
\hline 217 & L. liniaris T. Ander. & - & Acanthaceae & $\mathrm{h}$ \\
\hline 218 & Lesia spinosa Schott. & - & Araceae & $\mathrm{h}$ \\
\hline 219 & Leucas lavandulifolia Sm. & Gaochia & Lamiaceae & $\mathrm{h}$ \\
\hline 220 & Lindernia ciliata (Colms.) Pennel & - & Scrophulariaceae & $\mathrm{h}$ \\
\hline 221 & Lithocarpus elegans Hook. f. & Khami & Fagaceae & $\mathrm{t}$ \\
\hline 222 & Litsea glutinosa (Lour.) C. B. Robinson & Kukurchita & Lauraceae & $\mathrm{t}$ \\
\hline 223 & L. monopetala (Roxb.) Pers. & Akorma & Lauraceae & $\mathrm{t}$ \\
\hline 224 & Lophopetalum fimbriatum Wight. & Raktan & Celastraceae & $\mathrm{t}$ \\
\hline 225 & Macaranga denticulata (B1.) Muell.-Arg. & Bura & Euphorbiaceae & $\mathrm{s}$ \\
\hline 226 & M. indica Wight & - & Euphorbiaceae & $\mathrm{s}$ \\
\hline 227 & Maesa indica Wt. & Ramjoni & Myrsinaceae & $\mathrm{s}$ \\
\hline 228 & M. ramentacea Wall. & Maricha & Myrsinaceae & $\mathrm{s}$ \\
\hline 229 & Mallotus philippinensis (Lamk.). Muell.-Arg. & Punag & Euphorbiaceae & $\mathrm{s}$ \\
\hline 230 & M. roxburghii Muell.-Arg. & Nimputeli & Euphorbiaceae & $\mathrm{s}$ \\
\hline 231 & Mangifera indica L. & Aam & Anacardiaceae & $\mathrm{t}$ \\
\hline 232 & M. sylvatica Roxb. & Jangliam & Anacardiaceae & $\mathrm{t}$ \\
\hline 233 & Melastoma malabathricum Roxb. & Datrangan & Melastomaceae & $\mathrm{h}$ \\
\hline 234 & Melocalamus compactiflorus (Kurz) Benth. & Lotabans & Poaceae & $\mathrm{c}$ \\
\hline 235 & Melocana baccifera (Roxb.) Kurz & Mulibans & Poaceae & $\mathrm{s}$ \\
\hline 236 & Merremia umbellata (L.) Hallier f. & Sadakalmi & Convolvulaceae & $\mathrm{c}$ \\
\hline 237 & Michelia champaca L. & Champa & Magnoliaceae & $\mathrm{t}$ \\
\hline 238 & Micromelum minutum (Forst. f.) Wight \& Arn. & Bankunch & Rutaceae & $\mathrm{s}$ \\
\hline 239 & Mikania cordata (Burm. f.) B. L. Robinson & Assamlata & Asteraceae & $\mathrm{c}$ \\
\hline 240 & Mimosa intisia $\mathrm{L}$ & - & Mimosaceae & $\mathrm{s}$ \\
\hline 241 & M. pudica L. & Lajjabati & Mimosaceae & $\mathrm{h}$ \\
\hline 242 & Modecca trilobata Roxb. & - & Passifloraceae & $\mathrm{c}$ \\
\hline 243 & Monochoria hastata (L.) Solms. & Baranukha & Pontederiaceae & $\mathrm{h}$ \\
\hline 244 & Morinda angustifolia Roxb. & Ranggach & Rubiaceae & $\mathrm{s}$ \\
\hline 245 & Moringa oleifera Lamk. & Sajna & Moringaceae & $\mathrm{t}$ \\
\hline 246 & Mucuna monosperma DC. & Nataalkushi & Fabaceae & $\mathrm{c}$ \\
\hline 247 & M. pruiens (L.) DC. & Alkushi & Fabaceae & $\mathrm{c}$ \\
\hline 248 & Musa ornata Roxb. & Ramkola & Musaceae & $\mathrm{s}$ \\
\hline
\end{tabular}


Table 1 Contd.

\begin{tabular}{|c|c|c|c|c|}
\hline S1. N & Species name & Bengali name & Family & Habit \\
\hline 249 & M. paradisica $\mathrm{L}$. & Kachakola & Musaceae & $\mathrm{h}$ \\
\hline 250 & Mussaenda corymbosa Roxb. & Nagabali & Rubiaceae & $\mathrm{s}$ \\
\hline 251 & M. frondosa $\mathrm{L}$. & - & Rubiaceae & s \\
\hline 252 & Myхоругum smilacifolium B1. & - & Oleaceae & $\mathrm{h}$ \\
\hline 253 & Nelsonia canescens (Lamk.) Spreng. & - & Acanthaceae & $\mathrm{h}$ \\
\hline 254 & Olax nana Wall. & - & Olacaceae & $\mathrm{h}$ \\
\hline 255 & Ophiorrhiza harisiana Heyne & - & Rubiaceae & $\mathrm{h}$ \\
\hline 256 & O. villosa Roxb. & Ganjankuli & Rubiaceae & $\mathrm{h}$ \\
\hline 257 & Oplismenus burmanii (Retz.) P. Beauv. & - & Poaceae & $\mathrm{h}$ \\
\hline 258 & Ormosia robusta (Roxb.) Baker & - & Fabaceae & $\mathrm{t}$ \\
\hline 259 & Oroxyllum indicum (L.) Kurz & Thona & Bignoniacae & $\mathrm{t}$ \\
\hline 260 & Osbeckia rostrata D. Don & - & Melastomaceae & $\mathrm{h}$ \\
\hline 261 & Oxalis corniculata L. & Amrul & Oxalidaceae & $\mathrm{h}$ \\
\hline 262 & Paedaria foetida L. & Gandhabadhuli & Rubiaceae & $\mathrm{c}$ \\
\hline 263 & Pandanus foetidus Roxb. & Keyakanta & Pandanaceae & $\mathrm{s}$ \\
\hline 264 & Pavetta indica L. & Bisophal & Rubiaceae & $\mathrm{s}$ \\
\hline 265 & Peliosanthes teta Andr. & - & Haemodoraceae & $\mathrm{h}$ \\
\hline 266 & Persicaria hydropiper (L.) Spach. & Bishkatali & Polygonaceae & $\mathrm{h}$ \\
\hline 267 & P. minor (Huds) Opiz & - & Polygonaceae & $\mathrm{h}$ \\
\hline 268 & P. prosambu (Ham. ex D. Don) H. Gross & - & Polygonaceae & $\mathrm{h}$ \\
\hline 269 & P. strigosa (R. Br.) Nakai & - & Polygonaceae & $\mathrm{h}$ \\
\hline 270 & Phaulopsis imbricata (Forssk.) Sweet & - & Acanthaceae & $\mathrm{h}$ \\
\hline 271 & Phlogacanthus asperulus Nees & - & Acanthaceae & $\mathrm{h}$ \\
\hline 272 & P. curviflorous Nees & - & Acanthaceae & $\mathrm{h}$ \\
\hline 273 & P. tubiflorus Nees & - & Acanthaceae & $\mathrm{h}$ \\
\hline 274 & Phrynium imbricatum (Dietr.) Roxb. & Pitulpata & Marantaceae & $\mathrm{h}$ \\
\hline 275 & Phyllanthus amarus Schumacher \& Thonn. & - & Euphorbiaceae & $\mathrm{h}$ \\
\hline 276 & P. emblica L. & Amlaki & Euphorbiaceae & $\mathrm{t}$ \\
\hline 277 & P. reticulatus Poir. & Chitki & Euphorbiaceae & $\mathrm{s}$ \\
\hline 278 & P. sikkimensis Muell.-Arg. & - & Euphorbiaceae & $\mathrm{t}$ \\
\hline 279 & Pinanga gracilis $\mathrm{Bl}$. & Ramsupari & Arecaceae & $\mathrm{h}$ \\
\hline 280 & Piper betel L. & Pan & Piperaceae & $\mathrm{c}$ \\
\hline 281 & P. longum L. & Pepul & Piperaceae & $\mathrm{h}$ \\
\hline 282 & P. sylvaticum Roxb. & Paharipepul & Piperaceae & $\mathrm{c}$ \\
\hline 283 & Poganotherum panicum (Lamk.) Hack. & - & Poaceae & $\mathrm{h}$ \\
\hline 284 & Polygonum plebejum R. Br. & Anjaban & Polygonaceae & $\mathrm{h}$ \\
\hline 285 & Pothos scandens L. & Batilata & Araceae & $\mathrm{c}$ \\
\hline 286 & Premna esculenta Roxb. & Lallong & Verbenaceae & $\mathrm{s}$ \\
\hline 287 & Psidium guajava L. & Piara & Myrtaceae & $\mathrm{t}$ \\
\hline 288 & Psychotria fulva Ham. & - & Rubiaceae & $\mathrm{s}$ \\
\hline 289 & Pterospermum acerifolium Willd. & Kanakchampa & Sterculiaceae & $\mathrm{t}$ \\
\hline 290 & P. semisagittatum Ham. ex Roxb. & Banassar & Sterculiaceae & $\mathrm{s}$ \\
\hline
\end{tabular}


Table 1 Contd.

\begin{tabular}{|c|c|c|c|c|}
\hline S1. N & Species name & Bengali name & Family & Habit \\
\hline 291 & Quercus gomeziana A. Camus & - & Fagaceae & $\mathrm{t}$ \\
\hline 292 & Q. spicata Smith & Batna & Fagaceae & $\mathrm{t}$ \\
\hline 293 & Randia dumetorum Lamk. & Mankanta & Rubiaceae & $\mathrm{s}$ \\
\hline 294 & Rauvolfia serpentina (L.) Benth. ex Kurz & Sharpagandha & Apocynaceae & $\mathrm{h}$ \\
\hline 295 & Rhychoticum ellipticum A. DC. & - & Myrsinaceae & $\mathrm{s}$ \\
\hline 296 & Rubus hexagyna Roxb. & - & Rosaceae & $\mathrm{c}$ \\
\hline 297 & Rungia pectinata (L.) Nees & - & Acanthaceae & $\mathrm{h}$ \\
\hline 298 & Ryhnchostylis retusa (L.) B1. & - & Orchidaceae & $\mathrm{h}$ \\
\hline 299 & Saccharum arundanaceum Retz. & - & Poaceae & $\mathrm{h}$ \\
\hline 300 & S. spontaneum L. & Kash & Poaceae & $\mathrm{h}$ \\
\hline 301 & Sagittaria sagittifolia L. & Chottokut & Alismataceae & $\mathrm{h}$ \\
\hline 302 & Schima wallichii Choisy & Kanak & Theaceae & $\mathrm{t}$ \\
\hline 303 & Schizostachyum dulloa (Gamble) R. Majumdar & Dolu & Poaceae & $\mathrm{s}$ \\
\hline 304 & Scleria terrestris (L.) Fassett & - & Cyperaceae & $\mathrm{h}$ \\
\hline 305 & Scoparia dulcis L. & Bandhuni & Scrophulariaceae & $\mathrm{h}$ \\
\hline 306 & Setaria glauca (L.) P. Beauv. & Bajra & Poaceae & $\mathrm{h}$ \\
\hline 307 & Shorea robusta Gaertn. F. & Sal & Dipterocarpaceae & $\mathrm{t}$ \\
\hline 308 & Sida acuta Burm f. & Kureta & Malvaceae & $\mathrm{h}$ \\
\hline 309 & Smilax prolifera Roxb. & - & Smilacaceae & $\mathrm{c}$ \\
\hline 310 & S. zeylanica $\mathrm{L}$. & Kumarilata & Smilacaceae & $\mathrm{c}$ \\
\hline 311 & Solanum indicum $\mathrm{L}$. & Titbegun & Solanaceae & $\mathrm{s}$ \\
\hline 312 & S. torvum Sw. & - & Solanaceae & $\mathrm{h}$ \\
\hline 313 & Spilanthes acmella L. & Marhatitiga & Asteraceae & $\mathrm{h}$ \\
\hline 314 & Sporobolus diander (Retz) P. Beauv. & - & Poaceae & $\mathrm{h}$ \\
\hline 315 & S. indicus R. Br. & - & Poaceae & $\mathrm{h}$ \\
\hline 316 & Staurogyne argentea Wall. & - & Acanthaceae & $\mathrm{h}$ \\
\hline 317 & Stemona tuberosa Lour. & - & Stemonaceae & $\mathrm{c}$ \\
\hline 318 & Stephania harnandifolia Walp. & Muichanlata & Menispermaceae & $\mathrm{c}$ \\
\hline 319 & S. japonica (Thunb.) Miers. & Nimukha & Menispermaceae & $\mathrm{c}$ \\
\hline 320 & Sterculia colorata Roxb. & Udal & Sterculiaceae & $\mathrm{t}$ \\
\hline 321 & S. villosa Roxb. & Janlibadam & Sterculiaceae & $\mathrm{t}$ \\
\hline 322 & Steriospermum personatum (Hassk.) Chatt. & - & Bignoniacae & $\mathrm{t}$ \\
\hline 323 & Steudnera colocasioides Hook. f. & - & Araceae & $\mathrm{h}$ \\
\hline 324 & Stixis sauveolens Roxb. & - & Capparaceae & $\mathrm{c}$ \\
\hline 325 & Streblus asper Lour. & Shaora & Moraceae & $\mathrm{s}$ \\
\hline 326 & Strobilanthus scaber Nees & - & Acanthaceae & $\mathrm{h}$ \\
\hline 327 & Suregada multiflora (A. Juss.) Baill. & - & Euphorbiaceae & $\mathrm{s}$ \\
\hline 328 & Swietenia mahagoni (L.) Jacq. & Mehogoni & Meliaceae & $\mathrm{t}$ \\
\hline 329 & Synedrella nudiflora (L.) Gaertn. & - & Asteraceae & $\mathrm{h}$ \\
\hline 330 & Syzygium cumini (L.) Skeels & Kalojam & Myrtaceae & $\mathrm{t}$ \\
\hline 331 & S. firmum Thw. & Dhakijam & Myrtaceae & $\mathrm{t}$ \\
\hline 332 & S. formosanum (Hayata) Mor. & Panijam & Myrtaceae & $\mathrm{t}$ \\
\hline
\end{tabular}


Table 1 Contd.

\begin{tabular}{|c|c|c|c|c|}
\hline S1. N & Species name & Bengali name & Family & Habit \\
\hline 333 & S. fruticosum (Roxb.) DC. & Khudijam & Myrtaceae & $\mathrm{s}$ \\
\hline 334 & Tacca integrifolia Ker - Gawl. & Barahikand & Taccaceae & $\mathrm{h}$ \\
\hline 335 & Taxillus thelocarpa (Hook. f.) M. K. Alam & - & Loranthaceae & $\mathrm{s}$ \\
\hline 336 & Tectona grandis $\mathrm{L}$. & Segun & Verbenaceae & $\mathrm{t}$ \\
\hline 337 & Terminalia arjuna (Roxb. ex DC.) Wt. \& Arn. & Arjun & Combretaceae & $\mathrm{t}$ \\
\hline 338 & T. bellirica (Gaertn.) Roxb. & Bahera & Combretaceae & $\mathrm{t}$ \\
\hline 339 & T. citrina (Gaertn.) Roxb. ex Flaming & Hora & Combretaceae & $\mathrm{t}$ \\
\hline 340 & Tetasera sarmentosa (L.) Vahl. & - & Dilleniaceae & $\mathrm{c}$ \\
\hline 341 & Tetrameles nudiflora $\mathrm{R} . \mathrm{Br}$. & Tundul & Datiscaceae & $\mathrm{t}$ \\
\hline 342 & Tetrastigma thomsonianum Planch. & - & Vitaceae & $\mathrm{c}$ \\
\hline 343 & Thea sinensis L. & Cha & Theaceae & $\mathrm{s}$ \\
\hline 344 & Thladiantha cordifolia (B1.) Cogn. & - & Cucurbitaceae & $\mathrm{c}$ \\
\hline 345 & Thunbergia fragrans Roxb. & Nillata & Acanthaceae & $\mathrm{c}$ \\
\hline 346 & Thysanolena maxima (Roxb.) O. Kuntze & Phuljharu & Poaceae & $\mathrm{h}$ \\
\hline 347 & Tinospora cordifolia (Willd.) Hook. f. & Gulancha & Menispermaceae & $\mathrm{c}$ \\
\hline 348 & Toona ciliata M. Roem. & Toon & Meliaceae & $\mathrm{t}$ \\
\hline 349 & Torenia vagans Roxb. & - & Scrophulariacee & $\mathrm{h}$ \\
\hline 350 & Travesia palmata (Roxb.) Vis. & - & Araliaceae & $\mathrm{s}$ \\
\hline 351 & Trewia nudiflora L. & Pitali & Euphorbiaceae & $\mathrm{t}$ \\
\hline 352 & Trichosanthes bracteata (Lamk.) Voit. & - & Cucurbitaceae & $\mathrm{c}$ \\
\hline 353 & Triumfetta rhomboidea Jacq. & Banokra & Tiliaceae & $\mathrm{h}$ \\
\hline 354 & Uncaria sessilifructus Roxb. & - & Rubiaceae & $\mathrm{c}$ \\
\hline 355 & Uraria lagapoides DC. & - & Fabaceae & $\mathrm{h}$ \\
\hline 356 & Urena lobata L. & Banokra & Malvaceae & $\mathrm{h}$ \\
\hline 357 & Uvaria hamiltonii Hook. f. \& Thoms. & - & Annonaceae & $\mathrm{c}$ \\
\hline 358 & Vanda teres (Roxb.) Lindl. & - & Orchidaceae & $\mathrm{h}$ \\
\hline 359 & Vernonia cineria (L.) Lees. & Kuksim & Asteraceae & $\mathrm{h}$ \\
\hline 360 & $V$. extensa DC. & - & Asteraceae & $\mathrm{h}$ \\
\hline 361 & Vitex altissima L. & - & Verbenaceae & $\mathrm{t}$ \\
\hline 362 & V. glabrata R. Br. & Horina & Verbenaceae & $\mathrm{s}$ \\
\hline 363 & V. peduncularis Wall. ex Schauer & Awal & Verbenaceae & $\mathrm{t}$ \\
\hline 364 & Vitis latifolia Roxb. & Goalialata & Vitaceae & $\mathrm{c}$ \\
\hline 365 & $V$. trifolia $\mathrm{L}$ & Anallata & Vitaceae & $\mathrm{c}$ \\
\hline 366 & Wedelia trilobata (L.) A. S. Hitchc. & - & Asteraceae & $\mathrm{h}$ \\
\hline 367 & Willughbeia edulis Roxb. & Lata aam & Apocynaceae & $\mathrm{c}$ \\
\hline 368 & Xylia dolabiformis Benth. & Lohakat & Mimosaceae & $\mathrm{t}$ \\
\hline 369 & Zanthoxyllum rhetsa DC. & Bazna & Rutaceae & $\mathrm{t}$ \\
\hline 370 & Zingiber zerumbet (L.) Smith & Boj & Zingiberaceae & $\mathrm{h}$ \\
\hline 371 & Zizyphus mauritiana Lamk. & Boroi & Rhamnaceae & $\mathrm{s}$ \\
\hline 372 & Z. oenoplea (L.) Miller. & Banboroi & Rhamnaceae & $\mathrm{s}$ \\
\hline 373 & Z. oxyphylla Edgell & - & Rhamnaceae & $\mathrm{s}$ \\
\hline 374 & Z. xylophyrus (Retz.) Willd. & - & Rhamnaceae & $\mathrm{s}$ \\
\hline
\end{tabular}


The Park has few patches of natural forests, and plantations raised earlier by converting high forests of great biodiversity value. The top tree canopy includes Artocarpus chaplasha, Dipterocarpus turbinatus, Elaeocarpus floribundus, Dillenia pentagyna, Castanopsis tribuloides, Lophopetalum fimbriatum, Quercus spicata, Chukrassia tabularis, Ficus racemosa, Toona ciliata, Aphanamixis polystachia, Steriospermum personatum, Xylia dolabiformis, Lagerstroemia parviflora and Vitex peduncularis. The common shrub species are Micromelum minutum, Grewia microcos, Aphania danura, Erioglossum edulis, Macaranga peltata, Maesa indica, Travesia palmata, Carya arborea, Flacourtia indica, Randia dumetorum, Morinda angustifolia, Pavetta indica and Antidesma ghaesembila. The most common undergrowth species are mostly the members of Acanthaceae, Rubiaceae, Asteraceae, Poaceae, Cyperaceae, Zingiberaceae and Araceae. Most common climber species are the members of Acanthaceae, Apocynaceae, Asclepiadaceae, Asteraceae, Combretaceae, Convolvulaceae, Menispermaceae and Vitaceae. A luxuriant growth of epiphytes and parasites are observed on the forest trees. The most common epiphytes include Acampe premorsa, Aerides odorata, Dendrobium lindleyi and Vanda teres. The common bamboo species are Bambusa polymorpha, Bambusa tulda, Melocana baccifera and Schizostachyum dullooa. Valleys of the forest are often dominated by various members of Poaceae, Cyperaceae, Araceae, Polygonaceae, Zingiberaceae and Asteraceae.

Based on the field observations and present results it may be concluded that the angiosperm diversity of Lawachara National Park is very rich and the Park is the home for many threatened plant species of Bangladesh. The present result is a preliminary list of angiosperm diversity of the Park.

Currently plant diversity of this Park is in great risk because of many threats as observed during field works. Noteworthy threats are frequent forest fire during dry season, illegal logging, fire wood collections, betel leaf cultivation, oil exploration, development works, uncontrolled visitors and population pressure. Though the plant diversity of the park is under in situ conservation plan, the management plan should be made based on local knowledge of plant diversity. As the Park is the home of many threatened plant species and as well as for wildlife, for the sake of better management option distribution map of threatened plant species should be made on priority basis. Such map will facilitate accurate location and home range of threatened species in the Park so that monitoring activities can be carried out easily. In severe cases, ex situ conservation for particular species may be followed to replicate their population number. Present management system should be strengthened by deploying relevant manpower including plant taxonomists for proper documentation and conservation and sustainable development of Lawachara National Park. 


\section{Acknowledgement}

The authors are thankful to the authority of IUCN, Bangladesh and Forest Department for their cooperation during field visits of this study.

\section{References}

Ahmad, N. 1970. Working plan for the forests of the Sylhet division for the period 1963-64 to 1982-83, Working plan division 2, CTG.E.P. Dacca, East Pakistan Govt. Press. pp. 1-22.

Ahsan, M.F. 2000. Socio-Ecology of the Hoolock Gibbon (Hylobates hoolock) in two Forests of Bangladesh. Field Research. pp. 284-299.

Ahsan, M.M. 2007. Perceptions of tourism by indigenous communities living in and adjoining Lawachara National Park. In: Fox, J., Bushley, B.R, Dutt, S and Quazi, S.A. (eds.). Making conservation work: linking rural livelihoods and protected areas management in Bangladesh. East-West Centre and Nishorgo Program of the Bangladesh Forest Department. pp. 131-148.

Alam, M.K. 1988. Annotated checklist of the woody flora of Sylhet forests. Bull. 5. Plant Taxonomy Series. Bangladesh Forest Research Institute, Chittagong. 153 pp.

Alam, M.K. 1998. Documentation of Ethnobiological Information. In: Banik, R.L., Alam, M.K., Peil, S. and Rastogi, A. (eds.). Applied Ethnobotany. Bangladesh Forest Research Institute, Chittagong. pp. 28-29.

Canonizado, J.A. and Rahman, S.M. 1998. GOB/WB Forest resources management project technical asistance component: integrated forest management plan for the Sylhet forest division. Mandala Agricultural Development Corporation and Forest Department, Ministry of Environment and Forest, Dhaka, Bangladesh. pp. 1-4.

Green, 1990. IUCN Directory of South Asian protected areas. IUCN The Wrold Conservation Union, Gland, Switzerland and Cambridge, UK. pp. 24-30.

Khan, M.S., Rahman, M.M. and Ali, M.A. (eds.). 2001. Red Data Book of vascular plants of Bangladesh. Bangladesh National Herbarium. 179 pp.

Khan, M.S. and Huq, A.M. 2001. The vascular flora of Chunati Wildlife Sanctuary in south Chittagong, Bangladesh. Bangladesh J. Plant. Taxon. 8(1): 47-64.

Khan, M.S., Rahman, M.M., Huq, A.M., Mia, M.M.K. and Hassan M.A. 1994. Assesment of biodiversity of Teknaf game reserve in Bangladesh focusing on economically and ecologically important plants species. Bangladesh J. Plant. Taxon. 1(1): 21-33.

Rahman, M.O. and Hassan, M.A. 1995. Angiospermic flora of Bhawal National Park, Gazipur, Bangladesh. Bangladesh J. Plant Taxon. 2(1\&2): 47-79.

Riadh, S.M. 2007. Assessing the role of non-timber forest products in the livelihoods of communities living inside and outside of Lawachara National Park. In: Fox, J., Bushley, B.R, Dutt, S. and Quazi, S.A. (eds.). Making conservation work: linking rural livelihoods and protected areas management in Bangladesh. East-West Centre and Nishorgo Program of the Bangladesh Forest department. pp. 36-49.

Rizvi, S.N.H. 1970. East Pakistan District Gazetteers for Sylhet. Government of East Pakistan Survices and General Administration Department, Dhaka. pp. 5-6.

Stevens, P.R. 1986. Land classification and soil suitability for plantation in Sylhet division. Working Paper No. 26 (UNDP/FAO) Project BGD/79/017. 21 pp.

Uddin, M.Z. and Hassan, M.A. 2004. Flora of Rema-Kalenga Wildlife Sanctuary. IUCN Bangladesh Country Office, Dhaka, Bangladesh. 120 pp. 
Uddin, S.B. and Rahman, M.A. 1999. Angiospermic flora of Himchari National Park, Cox's Bazar. Bangladesh J. Plant Taxon. 6(1): 31-68.

Uddin, S.N., Khan, M.S., Hassan, M.A. and Alam, M.K. 1998. An annotated checklist of angiospermic flora of Sitapahar at Kaptai in Bangladesh. Bangladesh J. Plant Taxon. 5(1): 13-46.

(Manuscript received on 16 April 2009; revised on 3 August 2009) 